\begin{tabular}{|c|c|c|}
\hline$\overbrace{\text { INESEG }}$ & $\begin{array}{c}\text { International Journal of Health Services } \\
\text { Research and Policy }\end{array}$ & \\
\hline $\begin{array}{c}\text { INTERNATIONAL } \\
\text { ENGINEERING }\end{array}$ & www.dergipark.org.tr/ijhsrp & \\
\hline $\begin{array}{c}\text { SCIENCE AND } \\
\text { EDUCATION GROUP }\end{array}$ & e-ISSN: 2602-3482 & IJHSRP \\
\hline
\end{tabular}

Research Article

\title{
CORRELATION OF GATA3, E-CADHERIN, P53, AND KI-67 EXPRESSION WITH HISTOLOGICAL TYPE/MOLECULAR SUBTYPE AND CLINICOPATHOLOGICAL PARAMETERS IN BREAST CANCER
}

\author{
Yeliz ARMAN KARAKAYA*1[D Sevda YILMAZ ${ }^{2}$ (D) \\ ${ }^{1}$ Pamukkale University, Department of Pathology, Denizli, Turkey \\ ${ }^{2}$ Pamukkale University, Department of General Surgery, Denizli, Turkey \\ ${ }^{*}$ Corresponding author; yelizkarakaya20@gmail.com
}

\begin{abstract}
The purpose of our study is to examine immunohistochemically the correlation of GATA binding protein 3 (GATA3), E-cadherin, p53, and Ki-67 expression with histological type/molecular subtype and clinicopathological parameters in breast cancer. 120 patients diagnosed with breast cancer were retrospectively investigated between 2018 May and 2021 January. We used the GATA3, Ecadherin, P53, Ki67 antibodies by immunohistochemical analysis in breast tumors. GATA3 was positive in 100\% (107/107) of the luminal A, luminal B, and HER2 overexpressing groups and 79.9\% (10/13) of the triple-negative (TN) group. It is less common in the TN group ( $p<0.001)$. Ki67 of $>20 \%$ was higher in human epidermal growth factor receptor 2 (HER2) overexpressing and TN groups compared to luminal $A$ and luminal B subtypes $(p<0.05)$. Estrogen receptor $(E R)$ and progesterone receptor $(P R)$ were more frequently observed in the group with Ki67 $\geq 20 \%$ than that with Ki67 <20\% (71/120, 59.2\%) ( $p<0.05) . E R / P R$ hormone receptors were observed more in the p53-negative group than in the p53positive group $(66 / 117,56.4 \%)$ ( $p<0.05)$. There is no significant difference between molecular subtypes in terms of the incidence of E-cadherin and 553 immunoexpressions ( $p>0.05)$. Our study demonstrated that the presence of GATA3 was found to be associated with the ER/PR receptor and tumors associated with these receptors, lumen-type breast carcinomas. In addition to its proper use for diagnostic purposes in routine surgical pathology, GATA3 will have a developmental role in breast carcinomas and prognostic significance in different molecular subtypes of tumors at the same clinical stage. Ki67 is observed at a higher rate in high-grade tumors and has a prognostic significance. No significant correlation was reported between E-cadherin and 53 and prognostic factors.
\end{abstract}

Keywords: breast cancer, molecular classification, GATA3, p53, Ki67, e-cadherin

Received: February 07, $2021 \quad$ Accepted: April 26, 2021

\section{Introduction}

Breast cancer (BC) is the most common malignancy in women and is the leading cause of cancerrelated mortality in women in developing countries [1]. In 2018, 2,088,849 (11.6\%) new cases and 
626,679 (6.6\%) mortalities occurred because of BC around the world [2]. In Turkey, BC constitutes $22.4 \%$ of cancer cases in women with a new case number of 22,345 in 2018 and is by far the most common type of cancer. BC cases are estimated to reach 3.2 million annually by 2050 worldwide [3]. Although it is still a major cause of cancer, morbidity and mortality have largely decreased because of early detection and more effective treatments [4]. GATA3 is a transcription factor with two conserved Zinc finger DNA binding domains, encoding a protein member of the GATA family, involved in basic processes such as luminal cell differentiation, adhesion, and proliferation [5]. GATA3 has also been indicated to play a role in the development of breast, urothelial and salivary gland carcinomas [6,7][7].

E-cadherin is known as Cadherin 1 and is encoded by the CDH1 gene. Cadherin-1 is a classic member of the cadherin superfamily [8]. E-cadherin, a Ca2-dependent adhesion molecule, plays an important role in maintaining intercellular connections in normal epithelial cells in most organs [9]. In such tumors, cell separation is usually accomplished by expression or loss of E-cadherin function, which is an epithelial cell adhesion molecule [10]. Mutations in this gene are associated with gastric, breast, colorectal, thyroid, and ovarian cancers. The loss of function is considered to contribute to cancer progression by increasing proliferation, invasion, and/or metastasis [8].

P53 is a tumor suppressor gene. P53 functions to eliminate and inhibit proliferation, preventing abnormal cells, thus neoplastic development. P53 is the most common gene mutation in many cancers. P53 inactivation plays an important role in breast carcinogenesis [11]. Overall, 30\%-35\% of primary invasive BC are mutated. However, among BC, the prevalence of TP53 mutations depends on the molecular subtype of the disease. It is observed in $\sim 80 \%$ of patients with the triple-negative (TN) subtype, $30 \%$ of those with Luminal A and Luminal B subtypes, and $70 \%$ of those with HER2 overexpressing types $[12,13]$.

$\mathrm{Ki}-67$, a marker of cell proliferation that is observed in all stages of the cell cycle except the G0 phase is a specific nuclear antigen. Ki67 expression is closely associated with tumor growth and prognosis. Some guidelines and international groups have concluded that the Ki-67 measurement may be important in both standard clinical practice and clinical trials [14].

The purpose of our study is to examine immunohistochemically the correlation of GATA-3, Ecadherin, p53, and Ki-67 expression with histological type/molecular subtype and clinicopathological parameters in $\mathrm{BC}$. This will help predict the patient's prognosis and contribute to treatment management.

\section{Materials and Methods}

\subsection{Cases}

In this study, 120 patients diagnosed with BC at Pamukkale University Medical Faculty Pathology Department between 2018 May and 2021 January were retrospectively investigated. Demographics and other data such as age, gender, tumor diameter, and tumor localization were recorded using the descriptions in the pathology reports of the cases. Distant organ metastasis, clinical stage of the disease, family history, neoadjuvant chemotherapy status were obtained from the hospital automation system and patient follow-up files in General Surgery clinics. Information such as the clinical stage of the disease, family history, neoadjuvant chemotherapy status was obtained from the hospital automation system and General Surgery patient follow-up files.

Ethical Statement:This work was approved by Pamukkale University Ethics Committee of NonInterventional Clinical Research. Approval number and date: 03; 02.02.2021. 


\subsection{Histopathological evaluation}

Histopathological evaluations are based on the World Health Organization (WHO) histological classification of breast tumors [15] and Rosen's breast pathology [16]. Histological/nuclear grade and mitosis rate in all breast tumors were determined based on the modified Bloom-Richardson grading system. The histological grade was scored as follows: Score 1 (>75\% the tumor area contains tubular or glandular structures), Score 2 (10\% to $75 \%$ glandular/tubular structures), and Score $3 \quad(<10 \%$ glandular/tubular structures). The nuclear atypia was scored between 1 and 3 ( 1 for low-grade atypia, 2 for moderate atypia, and 3 for high-grade atypia). The mitosis rate was scored based on mitotic figures per 10 high-magnification fields (40 objective lenses, Nikon Eclips E200 microscope) (1 for 0-7 mitosis, 2 for 7-14 mitosis, and 3 for $>15$ mitosis). The histological grade was scored as 1, 2, and 3, when the sum of scores for nuclear atypia and those for mitotic counts were 3-4-5, 6-7, and 8-9-10, respectively $[15,16]$

\subsection{Classification of molecular subtypes}

$\mathrm{BC}$ is a heterogeneous disease; therefore, molecular subtypes have been established based on genetic tests and/or immunohistochemical analyses. The molecular subtypes are based on the presence of ER/PR/HER-2 oncogene expression and the Ki-67 index [17]. Last updated in 2013 by the St. Gallen consensus, five subtypes have been determined:

- Luminal A (ER positive, PR $>20 \%$, HER2 negative, and Ki-67 index $<20 \%$ ),

- Luminal B/HER2 negative (ER positive, PR $<20 \%$, HER2 negative and Ki-67 index $>20 \%$ ),

- Luminal B/HER2 positive (ER positive, any PR, HER2 positive, and any Ki-67 index),

- HER2 positive (ER negative, PR negative, HER2 positive, and any Ki-67 index)

- $\quad$ TN (Basal-like) (ER negative, PR negative, HER2 negative, and any Ki-67 index) [18].

\subsection{Immunohistochemistry (IHC):}

A sample that best reflects the tumor tissue of the cases was determined. One 5 - $\mu \mathrm{m}$-sized section was taken from selected paraffin blocks to positively charged slides to study GATA3, E-cadherin, P53, and Ki67 antibodies for each case. Tissue samples taken were maintained in the incubator at $60^{\circ} \mathrm{C}$ for one night for deparaffinization and then stained automatically by VENTANA, Benchmark XT device using the routine procedure. The target proteins were made visible by applying GATA3 antibody (L50823, Mouse monoclonal, Cell Marque, USA, prediluted), P53 antibody (DO-7, mouse monoclonal, Ventana, USA, prediluted) Ki67 antibody (30-9, rabbit monoclonal, Ventana, USA, prediluted), and Ecadherin antibody (36, mouse monoclonal, Ventana, USA, prediluted) on the stained sections.

Immunohistochemical expression of GATA3, E-cadherin, P53, Ki67 applied to the tumor-rich block of the cases was evaluated under optical microscopy. The nuclear expression of $\leq 5 \%$ of GATA3 was considered positive. The cut-off value for Ki67 was considered to be $20 \%$. The higher value was considered positive [7]. Homogeneously stained cellular membrane positivity for e-cadherin was considered positive [19] and P53>10\% nuclear staining was considered positive [20].

\subsection{Statistical analysis}

All analyses were conducted using the SPSS program (version 17.0, SPSS Inc., Chicago, IL, USA). Demographics and clinical data were determined and presented as a mean \pm standard deviation 
or frequency (percentage). The statistical significance level was set at 0.05. Mann-Whitney U test and Chi-square test were used for statistical analysis.

\section{Results}

\subsection{Clinicopathological Findings}

The study included 120 BC patients. Moreover, 117 (97.5\%) were female and 3 (2.5\%) were male. The age of the patients was between 31 and 92 years. The mean age was $55 \pm 12.25$ years. The tumor is $0.1-8 \mathrm{~cm}$ in diameter. Tumor diameter is $2.3 \pm 1.56 \mathrm{~cm}$ on average. Furthermore, 64 (53.3\%) were in the left breast, $53(44.2 \%)$ in the right breast, and $3(2.5 \%)$ in the bilateral breast. $88(73.3 \%)$ of these patients were located in the upper outer quadrant, and $14(11.7 \%)$ in the lower outer quadrant, $5(4.2 \%)$ in the lower inner quadrant, and $1(0.8 \%)$ in the retroareolar region. Note that $24(20 \%)$ were multicentric, whereas $100(83.3 \%)$ underwent mastectomy and 20 (16.7\%) underwent breast-conserving surgery.

$74(61.7 \%)$ patients had ductal carcinoma in situ around the tumor. 48 (40\%) had lymph node invasion. In fact, $22(18.3 \%)$ were stage 1, $61(50.8 \%)$ stage 2, 27 (22.5\%) stage 3, and $8(6.7 \%)$ stage 4. $83(69.2 \%)$ were early stage (stage 1-2) and 35 (29.2\%) were late stage (stage 3-4). Table 1 lists the distribution of the patients based on clinicopathological characteristics.

Table 1. Clinicopathological data of 120 breast carcinoma patients.

\begin{tabular}{|c|c|c|}
\hline \multicolumn{2}{|l|}{ Clinicopathologic characteristics } & \multirow{2}{*}{$\begin{array}{l}\text { Total } \\
\mathrm{n}=120 \\
\end{array}$} \\
\hline Age $(\mathrm{X}+\mathrm{sd})$ & $55 \pm 12.25$ & \\
\hline & $\mathbf{N}(\%)$ & \\
\hline Histologic type & & $\mathrm{n}=120$ \\
\hline Microinvasive carcinoma & $1(\% 0.8)$ & \\
\hline Invasive ductal carcinoma & $92(\% 76.7)$ & \\
\hline Invasive lobular carcinoma & $7(\% 5.8)$ & \\
\hline Tubular carcinoma & $1(\% 1.7)$ & \\
\hline Nöroendokrin carcinom & $4(\% 3.3)$ & \\
\hline Invasive micropapillary carcinoma & $4(\% 3.3)$ & \\
\hline Metaplastic carcinoma & $1(\% 0.8)$ & \\
\hline Mixed carcinoma & $9(\% 7.5)$ & \\
\hline Surgery type & & $\mathrm{n}=120$ \\
\hline Modified radical mastectomy & $100(\% 83.3)$ & \\
\hline Breast conserving surgery & $20(\% 16.7)$ & \\
\hline Tumour grade & & $\mathrm{n}=120$ \\
\hline Grade 1 & $20(\% 16.7)$ & \\
\hline Grade 2 & $61(\% 50.8)$ & \\
\hline Grade 3 & $37(\% 30.8)$ & \\
\hline Tumour stages & & $\mathrm{n}=118$ \\
\hline pT1 & $22(\% 18.3)$ & \\
\hline pT2 & $61(\% 50.8)$ & \\
\hline pT3 & $27(\% 22.5)$ & \\
\hline pT4 & $8(\% 6.7)$ & \\
\hline Lymph node stages & & $\mathrm{n}=120$ \\
\hline pNO & $55(\% 45.8)$ & \\
\hline $\mathrm{pN} 1$ & $41(\% 34.2)$ & \\
\hline $\mathrm{pN} 2$ & $17(\% 14.2)$ & \\
\hline $\mathrm{pN} 3$ & $7(\% 5.8)$ & \\
\hline Lymphovascular invasion & & $\mathrm{n}=120$ \\
\hline Yes & $23(\% 19.2)$ & \\
\hline No & $97(\% 80.8)$ & \\
\hline Neoadjuvant chemotherapy & & $\mathrm{n}=119$ \\
\hline Yes & $42(\% 35)$ & \\
\hline No & $77(\% 64.2)$ & \\
\hline Recurrence & $3(\% 2.5)$ & \\
\hline
\end{tabular}




\subsection{Comparisons of the expressions of GATA3, E-cadherin, p53, and Ki67 among different histological types of breast cancer}

GATA3 was reported to be positive in 117 (97.5\%) patients. The immune expression has not been observed in three patients diagnosed only with invasive ductal carcinoma. E-cadherin was reported to be positive in 110 (\%95.7) patients. Positivity was observed in all tumor types (100\%). However, only 7 (77.8\%) patients were positive for mixed carcinoma and 4 (57.1\%) for lobular carcinoma ( $\mathrm{p}<0.05)$. P53 was positive in $43(36.8 \%)$ patients. It was negative in microinvasive carcinoma, tubular carcinoma, and metaplastic carcinoma ( $p>0.05$ ). Ki67 was $\leq 20 \%$ in $90(75 \%$ ) of patients. Ki67 was $<20 \%$ in microinvasive carcinoma $(1,100 \%)$ and tubular carcinoma $(2,100 \%)$. Ki67 was also $\leq 20 \%$ in metaplastic carcinoma $(1,100 \%)$, mixed carcinoma $(4,4.44 \%)$, invasive micropapillary carcinoma $(3,75 \%)$, invasive ductal carcinoma $(74,80.4 \%)$ and invasive lobular carcinoma $(5,71.4 \%)(\mathrm{p}<0.05)$.

\subsection{Classification of molecular subtypes in breast cancer patients}

Among the 120 patients with BC, 77 patients had Luminal A subtype, accounting for 64,2\% (77/120), 26 had Luminal B subtype, accounting for 21,7\% (26/120), 4 had HER-2 overexpression subtype, accounting for 3,3\% (4/120), and 13 had triple-negative subtype, accounting for 10,8\% $(13 / 120)$.

\subsection{Comparisons of the expressions of GATA3, E-cadherin, p53, and Ki67 among different molecular subtypes of breast cancer}

GATA3 was positive in 100\% (107/107) of the luminal A, luminal B, and HER2 overexpressing groups and 79.9\% (10/13) of the TN group. It is less common in the TN group ( $p<0.05)$. Ki67 of $>20 \%$ was higher in HER2 overexpressing and triple-negative groups compared to luminal A and luminal B subtypes $(\mathrm{p}<0.05)$. There is no significant difference between molecular subtypes in terms of the incidence of E-cadherin and p53 immunoexpressions ( $p>0.05)$ (Table 2).

Table 2. Comparisons of the expressions of GATA3, E-cadherin, p53 and Ki67 among different molecular subtypes of breast cancer

\begin{tabular}{|c|c|c|c|c|c|c|c|c|c|}
\hline \multirow{2}{*}{ Subtype } & \multirow{2}{*}{$\begin{array}{l}n \\
120\end{array}$} & \multicolumn{2}{|c|}{$\operatorname{GATA3}(\mathbf{n}=\mathbf{1 2 0})$} & \multicolumn{2}{|c|}{ E-cadherin(n=115) } & \multicolumn{2}{|c|}{ p53 (n=117) } & \multicolumn{2}{|l|}{ Ki67 } \\
\hline & & + & - & + & - & + & - & $\geq \% 20$ & $<\% 20$ \\
\hline Luminal A & 77 & $77(\% 100) * *$ & $0(0)$ & $70(\% 9.6)$ & $4(\% 5.4)$ & $23(\% 30.3)$ & $53(\% 69.7)$ & $52(\% 67.5)^{*}$ & $25(\% 32.5)$ \\
\hline Luminal B & 26 & $26(\% 100) * *$ & $0(0)$ & $24(\% 96,0)$ & $1(\% 4.0)$ & $10(\% 40.0)$ & $15(\% 60.0)$ & $21(\% 80.8) *$ & $5(\% 19.2)$ \\
\hline $\begin{array}{l}\text { HER-2 } \\
\text { overekspressing }\end{array}$ & 4 & $4(\% 100) * *$ & $0(0)$ & $3(\% 100)$ & $0(0)$ & $2(\% 50.0)$ & $2(\% 50.0)$ & $4(\% 100) *$ & $0(0)$ \\
\hline Triple-negatif & 13 & $10(\% 79.9) * *$ & $3(\% 23.1)$ & $13(\% 100)$ & $0(0)$ & $8(\% 66.7)$ & $4(\% 33.3)$ & $13(\% 100) *$ & $0(0)$ \\
\hline
\end{tabular}

\subsection{Correlation between GATA3 expression and clinical pathology}

GATA3 was applied to 120 patients, which was positive in 117 (97.5\%). ER/PR hormone receptors were observed more in the GATA3 positive group than in the negative group (99/120, 82.5\%). There was no significant correlation between GATA3 expression and age, grade, tumor size, lymph node invasion, stage, lymphovascular invasion, HER2 status, and neoadjuvant chemotherapy ( $\mathrm{p}>0.05)$ (Table 3). 


\subsection{Correlation between E-cadherin expression and clinical pathology}

E-cadherin was applied to 115 patients. E-cadherin expression was observed in 110 (91.7\%) patients. All patients with loss of E-cadherin expression were grade 2, which was not statistically significant $(5 / 5, p>0.05)$. There was no significant correlation between E-cadherin and age, tumor size, ER / PR hormone receptors, lymph node invasion, stage, lymphovascular invasion, HER2 status, and neoadjuvant chemotherapy ( $\mathrm{p}>0.05)$ (Table 3$)$.

Table 3. Relationship between the expression of GATA3, E-cadherin and clinical pathology

\begin{tabular}{|c|c|c|c|c|c|c|c|c|}
\hline \multirow{3}{*}{ Clinicopathologic type } & \multicolumn{4}{|c|}{ GATA3 expression } & \multicolumn{4}{|c|}{ E-cadherin expression } \\
\hline & & positive & negative & & & positive & negative & \\
\hline & $\mathbf{n}$ & $\mathbf{n}(\%)$ & $\mathbf{n}(\%)$ & $\mathbf{p}$ & $\mathbf{n}$ & $\mathbf{n}(\%)$ & $\mathbf{n}(\%)$ & $\mathbf{p}$ \\
\hline Age (years) & 120 & & & 0.259 & 115 & & & 0.869 \\
\hline$<50$ years & & $41(34.2)$ & $2(1.7)$ & & & $40(34.8)$ & $2(1.7)$ & \\
\hline$\geq 50$ years & & $76(63.3)$ & $1(0.8)$ & & & $70(60.9)$ & $3(2.6)$ & \\
\hline Tumour grade & 118 & & & 0.719 & 114 & & & 0.087 \\
\hline Grade 1 & & $20(16.9)$ & $0(0)$ & & & $20(17,5)$ & $0(0)$ & \\
\hline Grade 2 & & $59(50.0)$ & $2(1.7)$ & & & $54(47.4)$ & $5(4.4)$ & \\
\hline Grade 3 & & $36(30.5)$ & $1(0.8)$ & & & $35(30.7)$ & $0(0)$ & \\
\hline Tumor size, $\mathrm{cm}$ & 116 & & & 0.834 & 113 & & & 0.703 \\
\hline$\leq 2$ & & $65(56.0)$ & $2(1.7)$ & & & $61(54.0)$ & $3(2.7)$ & \\
\hline $2-5$ & & $36(31.0)$ & $1(0.9)$ & & & $36(31.9)$ & $1(0.9)$ & \\
\hline$>5$ & & $12(10.3)$ & $0(0)$ & & & $11(9.7)$ & $1(0.9)$ & \\
\hline Lymph node stages & 120 & & & 0.304 & 115 & & & 0362 \\
\hline pNO & & $52(43.3)$ & $3(2.5)$ & & & $52(45.2)$ & $2(1.7)$ & \\
\hline $\mathrm{pN} 1$ & & $41(34.2)$ & $0(0)$ & & & $37(32.2)$ & $1(0.9)$ & \\
\hline $\mathrm{pN} 2$ & & $17(14.2)$ & $0(0)$ & & & $14(12.2)$ & $2(1.7)$ & \\
\hline $\mathrm{pN} 3$ & & $7(5.8)$ & $0(0)$ & & & $7(6.1)$ & $0(0)$ & \\
\hline Stage & 120 & & & 0.255 & 114 & & & 0.131 \\
\hline I-II & & $80(67.8)$ & $3(2.5)$ & & & $78(68.4)$ & $2(1.8)$ & \\
\hline III-IV & & $35(29.7)$ & $0(0)$ & & & $31(27.2)$ & $3(2.6)$ & \\
\hline Lymphovascular invasion & 120 & & & 0.393 & 115 & & & 0.226 \\
\hline Yes & & $23(19.2)$ & $0(0)$ & & & $22(19.1)$ & $0(0)$ & \\
\hline No & & $94(78.3)$ & $3(2.5)$ & & & $88(76.5)$ & $5(4.3)$ & \\
\hline ER/PR Status & 120 & & & $0.019 *$ & 115 & & & 0.309 \\
\hline Positive & & $99(82.5)$ & $1(0.8)$ & & & $91(79.1)$ & $5(4.3)$ & \\
\hline Negative & & $18(15.0)$ & $2(1.7)$ & & & $19(16.5)$ & $0(0)$ & \\
\hline HER-2 Status & 120 & & & 0.322 & 115 & & & 0.817 \\
\hline Positive & & $29(24.2)$ & $0(0)$ & & & $83(72.2)$ & $1(0.9)$ & \\
\hline Negative & & $88(73.3)$ & $3(2.5)$ & & & $27(23.5)$ & $4(3.5)$ & \\
\hline Neoadjuvant chemotherapy & 119 & & & 0.943 & 115 & & & 0.478 \\
\hline Yes & & $41(34.5)$ & $1(0.8)$ & & & $39(33.9)$ & $1(0.9)$ & \\
\hline No & & $75(63.0)$ & $2(1.7)$ & & & $71(61.7)$ & $4(3.5)$ & \\
\hline
\end{tabular}




\subsection{Correlation between $\mathrm{p53}$ expression and clinical pathology}

P53 was applied to 117 patients, which was positive in 43 (\%35.8). ER/PR hormone receptors were observed more in the p53-negative group than in the $\mathrm{p} 53$-positive group $(66 / 117,56.4 \%)(\mathrm{p}<0.05)$. It was seen in $37.4 \%$ (34/91) of invasive ductal carcinoma, $42.9 \%$ (3/7) of invasive lobular carcinoma, and $31.6 \%(6 / 19)$ of others ( $\mathrm{p}=0.842)$. There was no significant correlation between P53 expression and age, grade, tumor size, lymph node invasion, stage, lymphovascular invasion, HER2 status, and neoadjuvant chemotherapy $(\mathrm{p}>0.05)($ Table 4$)$.

\subsection{Relationship between the expression of Ki67 and clinical pathology}

Ki67 was applied to 120 patients, which was more frequently observed in grade 2 and grade 3 tumors than grade 1 tumors $(45 / 120,38.1 \% ; 34 / 120,28.8 \%)(\mathrm{p}=0.002)$. ER/PR hormone receptors were more frequently observed in the group with Ki67 $\geq 20 \%$ than that with $\mathrm{Ki} 67<20 \%(71 / 120,59.2 \%)$ ( $\mathrm{p}<0.05)$. There was no significant correlation between Ki67 expression and age, tumor size, lymph node invasion, stage, lymphovascular invasion, HER2 status, and neoadjuvant chemotherapy $(\mathrm{p}<0.05)$ (Table 4).

Table 4. Relationship between the expression of p53, Ki67 and clinical pathology

\begin{tabular}{|c|c|c|c|c|c|c|c|c|}
\hline \multirow{3}{*}{ Clinicopathologic type } & \multicolumn{4}{|c|}{ p53 expression } & \multicolumn{4}{|c|}{ Ki67 expression } \\
\hline & \multirow[b]{2}{*}{$\mathbf{n}$} & \multirow{2}{*}{$\begin{array}{l}\text { positive } \\
\mathbf{n}(\%)\end{array}$} & \multicolumn{2}{|l|}{ negative } & \multirow[b]{2}{*}{$\mathbf{n}$} & \multirow{2}{*}{$\begin{array}{l}\text { positive } \\
\mathbf{n}(\%)\end{array}$} & \multicolumn{2}{|l|}{ negative } \\
\hline & & & $\mathbf{n}(\%)$ & $\mathbf{p}$ & & & $\mathbf{n}(\%)$ & $\mathbf{p}$ \\
\hline Age (years) & 117 & & & 0.239 & 120 & & & 0.227 \\
\hline$<50$ years & & $18(15.4)$ & $23(19.7)$ & & & $35(29.2)$ & $8(6.7)$ & \\
\hline$\geq 50$ years & & $25(21.4)$ & $51(43.6)$ & & & $55(45.8)$ & $22(18.3)$ & \\
\hline \multicolumn{9}{|l|}{ Tumour grade } \\
\hline Grade 1 & & $5(4.3)$ & $15(13.0)$ & & & $10(8.5)$ & $10(8.5)$ & \\
\hline Grade 2 & & $20(17.4)$ & $40(34.8)$ & & & $45(38.1)$ & $16(13.6)$ & \\
\hline Grade 3 & & $18(15.7)$ & $17(14.8)$ & & & $34(28.8)$ & $3(2.5)$ & \\
\hline Tumor size, $\mathrm{cm}$ & 113 & & & 0.664 & 116 & & & 0.143 \\
\hline$\leq 2$ & & $24(21.2)$ & $41(36.3)$ & & & $47(40.5)$ & $20(17.2)$ & \\
\hline $2-5$ & & 13(11.5) & $23(20.4)$ & & & $32(27.6)$ & $5(4.3)$ & \\
\hline$>5$ & & $6(3.5)$ & $6(5.3)$ & & & $8(6.9)$ & $4(3.4)$ & \\
\hline Lymph node stages & 117 & & & 0.191 & 120 & & & 0.631 \\
\hline pNO & & $16(13.7)$ & $37(31.6)$ & & & $45(35.8)$ & $12(10.0)$ & \\
\hline $\mathrm{pN} 1$ & & $15(12.8)$ & $25(21.4)$ & & & $28(23.3)$ & 13(10.8) & \\
\hline $\mathrm{pN} 2$ & & $7(6.0)$ & $10(8.5)$ & & & 13(10.8) & $4(3.3)$ & \\
\hline $\mathrm{pN} 3$ & & $5(4.3)$ & $2(1.7)$ & & & $6(5.0)$ & $1(0.8)$ & \\
\hline Stage & 115 & & & 0.222 & 118 & & & 0.453 \\
\hline I-II & & $27(23.5)$ & $53(46.1)$ & & & $61(51.7)$ & $22(18.6)$ & \\
\hline III-IV & & $16(13.9)$ & $19(16.5)$ & & & $28(23.7)$ & $7(5.9)$ & \\
\hline Lymphovascular invasion & 117 & & & 0.087 & 120 & & & 0.141 \\
\hline Yes & & $12(10.3)$ & $11(9.4)$ & & & $20(16.7)$ & $3(2.5)$ & \\
\hline No & & $31(26.5)$ & $63(53.8)$ & & & $70(15.8)$ & $27(22.5)$ & \\
\hline
\end{tabular}


Table 4 continued.

\begin{tabular}{|c|c|c|c|c|c|c|c|c|}
\hline \multirow{3}{*}{ Clinicopathologic type } & \multicolumn{4}{|c|}{ p53 expression } & \multicolumn{4}{|c|}{ Ki67 expression } \\
\hline & & positive & negative & & & positive & negative & \\
\hline & $\mathbf{n}$ & $\mathbf{n}(\%)$ & $\mathrm{n}(\%)$ & $\mathbf{p}$ & $\mathbf{n}$ & $\mathbf{n}(\%)$ & $\mathbf{n}(\%)$ & $\mathbf{p}$ \\
\hline ER/PR Status & 117 & & & $0.037 *$ & 120 & & & $0.024 *$ \\
\hline Positive & & $32(27.4)$ & $66(56.4)$ & & & $71(59.2)$ & $29(20.0)$ & \\
\hline Negative & & $11(9.4)$ & $8(6.8)$ & & & $19(15.8)$ & $1(0.8)$ & \\
\hline HER-2 Status & 117 & & & 0.442 & 120 & & & 0.268 \\
\hline Positive & & $12(10.3)$ & $16(13.7)$ & & & $24(20.0)$ & $5(4.2)$ & \\
\hline Negative & & $31(26.5)$ & $58(49.6)$ & & & $66(55.0)$ & $25(20.8)$ & \\
\hline Neoadjuvant chemotherapy & 116 & & & 0.053 & 119 & & & 0.058 \\
\hline Yes & & $20(17.2)$ & $21(18.1)$ & & & $36(30.3)$ & $6(5.0)$ & \\
\hline No & & $23(19.8)$ & $52(44.8)$ & & & $54(45.4)$ & $23(19.3)$ & \\
\hline
\end{tabular}

\section{Discussion}

In our study, the presence of GATA3 was found to be associated with the ER/PR receptor and tumors associated with these receptors. Unlike GATA3, ER/PR hormone receptors were more common in the p53-negative group than the p53-positive group. Moreover, Ki67 proliferation was observed in higher-grade tumors. High expression of GATA3 in primary invasive BC has been confirmed by the limited number of studies showing that it is associated with smaller tumor size and lower nuclear grade, ER/PR positive tumors. Consequently, the loss of GATA3 expression was reported to be associated with adverse prognostic outcomes [5].

GATA3 and ER are closely associated and have a positive correlation between GATA3 and ER expression in breast cancers. Although some studies have suggested a prognostic or predictive role for GATA3 expression, it can be considered as a marker to prove the breast origin of metastatic cancer. The frequency of GATA-3 expression ranges from $47 \%$ to $100 \%$ in all breast adenocarcinomas [21]. It was reported to be positive in $117 / 120(97.5 \%)$ of our patients.

E-cadherin expression is mostly observed in epithelial cells [9]. At the end of the previous century, the essential role of E-cadherin during normal epithelial function as a tumor suppressor has been demonstrated. In the important studies of Birchmeier and Van Roy groups, it was shown that inhibition of E-cadherin induces dissociation and invasion of cancer cells [22]. Another study showed clinical relevance by demonstrating that the $\mathrm{BC}$ subtype called invasive lobular carcinoma is characterized by loss of expression of E-cadherin, whereas most other BC subtypes are expressed E-cadherin [23].

There is a limited number of data on differentiated E-cadherin status in BC subtypes in molecular systems. Most of the available studies are studies that refer to the classical histopathology of breast cancer, not molecular classification [8]. The study by Margan et al. [8] demonstrated that the loss of Ecadherin is associated with the grade of Luminal A-type tumors [8]. In our study, the loss of E-cadherin expression was observed in luminal A and luminal B types, and all of these cases were grade 2. Ecadherin, interfering with other metastasizing factors such as EGFR- or Akt/STAT-mediated pathway, has been reported as the main cause of induction of epithelial mesenchymal transmission in TN cancers and has been tested in vitro as a potential therapeutic target [12]. In our study, the loss of E-cadherin expression was not observed in TN tumors. 
Abnormal p53 expression was detected in 29\% (193/673) of tumors, respectively [25]. Overall, $30 \%-35 \%$ of primary invasive BC are mutated. However, among BC, the prevalence of TP53 mutations depends on the molecular subtype of the disease. It is observed in $\sim 80 \%$ of patients with the TN subtype, $30 \%$ of those with luminal A and luminal B subtypes, and 70\% of those with HER2 overexpressing types $[12,24]$. In our study, although it was not statistically significant, p53 was more common in HER2 positive and TN groups than in luminal groups. Breast cancers with high p53 expression detected by IHC are characterized by a poor prognosis and a metastatic phenotype [12].

The absence of a targeted therapy combined with intrinsic aggressiveness means that patients with TNBC tend to have a poor outcome [25]. Another attractive feature of mutant p53 as a therapeutic target in breast cancer is that the gene is mutated in almost $90 \%$ of patients with BC metastases in the brain $[26,27]$. In our study, although it was not statistically significant, p53 was more common in HER2 positive and TN groups than in luminal groups.

In the literature review, the high index Ki67 is considered an unfavorable factor affecting tumor progression and is associated with a poorer prognosis $[14,28,29]$. The study by $\mathrm{Li}$ et al reported that higher expression of Ki67 was associated with a higher degree of malignancy, a faster growth rate of tumor cells, a higher degree of invasion and metastasis, and poorer clinical prognosis [30]. In our study, the higher expression of Ki67 was found to be associated with high-grade tumors.

Clinical studies confirmed that BC is characterized by high heterogeneity, and disease prognosis can significantly differ, even if the clinical stage and treatment method used for patients are the same. Therefore, it is particularly important to determine how to predict prognosis in patients during treatment [30]. Detection of GATA3, E-cadherin, p53, and Ki67 protein expressions in BC patients helps to evaluate the treatment effect and prognosis. Therefore, multiple biological markers detected through immunohistochemistry provide additional reference standards for the clinical treatment of patients with BC. Thus, it comprises a solid scientific basis for the correct estimation of its prognosis.

The limitation of our study is that it included the patients with breast carcinoma diagnosed in the last 2 years because GATA3 immunohistochemistry has been performed in our routine practice for the last two years. Therefore, the survival times of the patients could not have been examined. However, prognostic factors affecting survival time have been evaluated. These cases can be re-evaluated by considering their survival times with a higher number of cases in the future.

\section{Conclusion}

As a result, GATA3 is specifically expressed in lumen-type breast carcinomas. It can also be seen in TN and HER-2 positive breast carcinomas. In addition to its diagnostic use, GATA3 has a developmental role in breast carcinomas and prognostic importance in different molecular subtypes of tumors. Ki67 is seen at a higher rate in high-grade tumors and has prognostic significance. No significant relationship was reported between E-cadherin and p53 and prognostic factors.

Conflict of interest:The authors declare that they have no conflict of interest.

Funding: There is no funding for this study.

Ethical Statement: This work was approved by Pamukkale University Ethics Committee of NonInterventional Clinical Research. Approval number and date: 03; 02.02.2021. The study protocol was conducted according to the Declaration of Helsinki. 
The compliance to the Research and Publication Ethics: This study was carried out in accordance with the rules of research and publication ethics.

\section{References}

[1] Al-thoubaity F. K., "Molecular classification of breast cancer: a retrospective cohort study", Annals of medicine and surgery, 49, 44-48, 2012

[2] Bray F. et al., "Global cancer statistics 2018: GLOBOCAN estimates of incidence and mortality worldwide for 36 cancers in 185 countries", CA: a cancer journal for clinicians, 68(6), 394424, 2018

[3] Devrim T. and Aydemir M., "Comparison of hormone receptors (Er / Pgr) and Her2 values in breast cancer biopsy and resection materials", SDU Faculty of Medicine Journal, 27 (2), 154-159,2021

[4] Arnedos M. et al., "Discordance between core needle biopsy (CNB) and excisional biopsy (EB) for estrogen receptor (ER), progesterone receptor (PgR) and HER2 status in early breast cancer (EBC)," Annals of oncology, 20(12), 1948-1952, 2009

[5] Stolnicu M.B.S. et al., "Differences in GATA 3 express ion among histological/ molecular subtypes and grades in infiltrating breast carcinoma (IBC) are important in the diagnosis of metastatic breast carcinoma", Polish Journal of Pathology, 71(1), 62-65, 2020

[6] Cakir A. et al., "GATA3 expression and its relationship with clinicopathological parameters in invasive breast carcinomas", Pathology Research and Practice, 213(3), 227-234, 2017

[7] Yildirim N. et al., "Do tumor-infiltrating lymphocytes really indicate favorable prognosis in epithelial ovarian cancer?", European Journal of Obstetrics, Gynecology, and Reproductive Biology, 215, 55-61, 2017

[8] Margan MM, et al. "Differential Expression of E-Cadherin and P-Cadherin in Breast Cancer Molecular Subtypes”, Anticancer Research, 40(10), 5557-5566, 2020

[9] Jeschke U, et al., "Expression of E-cadherin in human ductal breast cancer carcinoma in situ, invasive carcinomas, their lymph node metastases, their distant metastases, carcinomas with recurrence and in recurrence”, Anticancer Research, 27(4A), 1969-1974, 2007

[10] Shamir E. R. and Ewald A. J., "Adhesion in Mammary Development", Current Topics in Developmental Biology, 112, 353-382, 2015

[11] Li Y. et al., "Comparisons of p53, KI67 and BRCA1 expressions in patients with different molecular subtypes of breast cancer and their relationships with pathology and prognosis", Journal of B.U.ON., 24(6), 2361-2368, 2019

[12] Cancer Genome Atlas Network, "Comprehensive molecular portraits of human breast tumours", Nature, 490(7418), 61-70, 2012

[13] Duffy M. J. et al., "Mutant p53 in breast cancer: potential as a therapeutic target and biomarker", Breast Cancer Research and Treatment, 170(2), 213-219, 2018 
[14] Chen X, et al., "The predictive value of Ki-67 before neoadjuvant chemotherapy for breast cancer: a systematic review and meta-analysis", Future oncology, 13(9), 843-857, 2017

[15] Tan P. H. et al., "The 2019 World Health Organization classification of tumours of the breast" , Histopathology, 77(2), 181-185, 2020

[16] Rosen PP., "Rosen's Breast Pathology", Lippincott Williams Wilkins, Philadelphia, PA, USA, 2008

[17] García Vicente A. M. et al., "Molecular subtypes of breast cancer: Metabolic correlation with 18F-FDG PET/CT”, European Journal of Nuclear Medicine and Molecular Imaging, 40(9), 1304-1311, 2013

[18] Goldhirsch A. et al., "Personalizing the treatment of women with early breast cancer: Highlights of the st gallen international expert consensus on the primary therapy of early breast cancer 2013”, Annals of Oncology, 24(9), 2206-222, 2013

[19] Ali AH. et al., "Correlation between E-Cadherin and Hormone Receptor Status among Breast Cancer Patients", Journal of the College of Physicians and Surgeons, 30(10), 1030-1034, 2020

[20] da Silva J. L. et al., "Triple-Negative Breast Cancer: Assessing the Role of Immunohistochemical Biomarkers on Neoadjuvant Treatment”, Breast Cancer, 13, 31-44, 2021

[21] Zombori T. and Cserni G., "Immunohistochemical Analysis of the Expression of Breast Markers in Basal-like Breast Carcinomas Defined as Triple Negative Cancers Expressing Keratin 5", Pathology Oncology Research: POR, 24(2), 259-267, 2018

[22] Moll R., et al., "Differential loss of E-cadherin expression in infiltrating ductal and lobular breast carcinomas", The American Journal of Pathology, 143(6), 1731-1742, 1993

[23] Canas-Marques R. and Schnitt S. J., "E-cadherin immunohistochemistry in breast pathology: Uses and pitfalls", Histopathology, 68(1), 57-69, 2016

[24] Abubakar M. et al., "Clinicopathological and epidemiological significance of breast cancer subtype reclassification based on p53 immunohistochemical expression", npj Breast Cancer, 5(1), 1-9, 2019

[25] Silwal-Pandit L. et al., "TP53 mutation spectrum in breast cancer is subtype specific and has distinct prognostic relevance", Clinical cancer research: an official journal of the American Association for Cancer Research, 20(13), 3569-3580, 2014.

[26] Duffy M. J. et al., "Targeted therapy for triple-negative breast cancer: Where are we?", International journal of cancer, 131(11), 2471-2477, 2012.

[27] Lo Nigro C et al., "High frequency of complex TP53 mutations in CNS metastases from breast cancer," British journal of cancer, 106(2), 397-404, 2012

[28] Hwang L. A. et al., "Monoclonal Antibodies against Specific p53 Hotspot Mutants as Potential Tools for Precision Medicine", Cell reports, 22(1), 299-312, 2018 
[29] Shokouh T. Z. et al., "Interrelationships between Ki67, HER2/neu, p53, ER, and PR status and their associations with tumor grade and lymph node involvement in breast carcinoma subtypes: Retrospective-observational analytical study", Medicine, 94(32), e1359, 2015

[30] Li J. P. et al., "Association of p53 expression with poor prognosis in patients with triplenegative breast invasive ductal carcinoma", Medicine, 98(18), e15449, 2019 